\title{
Analysis of Rescued Learning with Different Drugs in down Syndrome
}

\author{
Handan Kulan*, Tamer Dağ \\ Computer Engineering Department, Kadir Has University, Istanbul, Turkey. \\ * Corresponding author. Email: 20161102001@stu.khas.edu.tr \\ Manuscript submitted September 19, 2018; accepted January 12, 2019. \\ doi: 10.17706/ijbbb.2019.9.3.173-179
}

\begin{abstract}
Down syndrome (DS) affects approximately one in 700 live births and it is considered as the most prevalent cause of intellectual disability (ID). DS is caused by the presence of an extra copy of the human chromosome21 (Hsa21) and has been investigated on protein levels by using the Ts65Dn mouse model. For the treatment of DS, many efforts have been made for developing drugs to rescue learning performance. In this paper, we apply forward feature selection method to identify the important proteins in which memantine and GABAA alpha5 receptor inverse agonist- R04938581 drugs affect. Identifying these gene products will help researchers to determine the molecular pathways which play key roles for rescuing performance in DS. Analyzing these pathways helps us to not only understand the learning process but also to contribute the new drug design for the treatment of DS.
\end{abstract}

Key words: Down syndrome, protein expression, memantine, R04938581.

\section{Introduction}

DS is caused by trisomy of Hsa21 and the increased expression, due to dosage, of some subset of the encoded genes [1]. The overexpression of genes encoded by the extra copy of Hsa21 in DS is believed to be sufficient to perturb many different biological processes and pathways, such as affecting brain development and causing learning and memory deficits [2]. Individuals with DS show some level of ID and delay in motor skill development. Most individuals achieve major life skills on their own developmental course, and show communicative intent, regardless of limitations in their verbal ability [3], [4].

There are no pharmacotherapies for learning deficits in DS. Due to the severity and the high incidence rate of DS, researchers have used mice for the development of cure for DS. However, DS is challenging to model in mice as orthologs of Hsa21 genes map to segments of three mouse chromosomes, indicated as Mmu16, Mmu17, and Mmu10. The Ts65Dn mouse type is the first viable segmental trisomy mouse model for DS. It is trisomic for 55\% of Hsa21 orthologous protein-coding genes and is also trisomic for a large number of genes that are not orthologs of Hsa21 proteins. The Ts65Dn displays many features relevant to DS, including abnormalities in neuronal number and morphology in some brain regions and deficits in learning and memory (L/M) [5], [6].

Over the last few years, numerous drugs and small molecules have been shown to rescue one or more abnormalities in the Ts65Dn [7]-[15]. Untreated Ts65Dn mice fail to learn but if they are first injected with drug, they can learn successfully, i.e., learning is rescued. These successes have led to considerable enthusiasm for clinical trials to develop drugs. One of the drugs is memantine and it is currently in use for 
treatment of learning deficits in DS to rescue performance in several L/M tasks. While memantine is known to modulate excitatory neurotransmission through antagonizing activity of Nmethyl-D-aspartate (NMDA) receptor, little is known about its effects on protein expression, either alone or with learning paradigms [16], [17].

Increased GABAA-mediated inhibition has been proposed to be a crucial mechanism for contributing to the L/M alterations found in the Ts65Dn. Another drug, R04938581 (3- bromo-10-(difluoromethyl)9H-benzo[f]imidazo[1,5-a][1,2,4] triazolo [1,5-d] [1,4] diazepine) is GABAA receptor negative allosteric modulator (NAM), with dual binding and functional affinity specific for GABAA receptors containing the a5 subunit. Like memantine, it is also used for rescuing protein anomalies in the Ts65Dn. It has been shown that R04938581 improves L/M and rescue neurogenesis, without the side effects, such as anxiety and convulsions [9].

Protein functions and possible interactions between them and drugs occur differentially in people with DS. Comparison of protein profiles between the Ts65Dn when they fail and the Ts65Dn when their learning is rescued with drugs reveal statistically significant changes in protein levels associated with rescued learning.

Rescued performance of drugs in DS has been studied in the literature. Ahmed et al. [18] performed a three level mixed effects (3LME) statistical analysis model of the trisomic and normal mice protein profiles with and without exposure to memantine. They showed that more than half of the protein levels change significantly in the hippocampus when exposed to either contextual fear conditioning (CFC) or memantine and there were significant differences between the profiles of normal and Ts65Dn mice. However, the standard statistical analyses employed in the Ts65Dn studies do not identify several important features, for example, which changes induced by drug are critical for rescuing learning in the Ts65Dn. The machine learning methods might fulfill these needs. Thus, Higuera et al. [19] analyzed the profiles using unsupervised learning, Self Organizing Map (SOM) to find the discriminant proteins in rescued learning with drug memantine. They used the Wilcoxon rank-sum test to identify proteins whose levels are significantly different between classes of mice. They analyzed expression levels of 77 proteins obtained from normal genotype control mice and from their trisomic littermates (Ts65Dn) both with and without treatment with the memantine drug. With the selected proteins, mice in failed learning discriminate from mice in rescued learning efficiently. Also, Block et al [9] used GABAA $\alpha 5$ selective modulator, R04938581, for rescuing protein anomalies in the Ts65Dn.

In their work, 91 protein levels relevant to brain functions were measured by applying the 3LME and 44 of the 52 anomalies in Ts65Dn were corrected by R04938581. In this work, the forward feature selection method is applied for selecting subset of features from protein expression datasets which comprise memantine and R04938581 drugs interactions between proteins. These selected protein subsets which show the critical factors for differentiating mice in rescued and failed learning are compared in order to understand effects of drugs on protein expression. This type of analysis can help to address questions such as whether different drugs-treated Ts65Dn mice exhibit similar response or not.

In this research, we demonstrate that two drugs, memantine and R04938581, affect the common proteins. It can be utilized from this outcome to understand the effects of drugs on DS and effective drugs can be developed.

The rest of paper is designed as follows; section two explains the materials and methods part. Section three shows the selected protein subsets of different drugs treated Ts65Dn mice and discuss the results. In the conclusion section, we evaluate the response of different drugs on mice protein expression and contribution of obtained information from these responses to diagnose and cure the DS. 


\section{Material and Method}

\subsection{Datasets}

In this work, two datasets are used. The dataset of memantine drug which contains a total of 1080 measurements per protein is taken from the Machine Learning Repository at University of California Irvine [20]. It consists of the expression levels of 77 protein modifications that produced detectable signals in the nuclear fraction of the cortex. Protein lysates were prepared from the brains of three month old male Ts65Dn DS model mice and their male littermate wildtype controls. There are 38 control mice and 34 trisomic mice. 15 samples, three replicates of a five-point dilution series, are obtained per mouse, adding up to a total of 1080 samples. The dataset is divided into eight classes of mice based on protein profiles of 77 proteins after training in CFC with and without injection of memantine. The eight classes of mice are described based on features such as genotype, behavior and treatment. With CFC, data of failed and rescued learning classes are determined from the protein expression response of seven and nine mice, respectively.

The dataset of R04938581 drug is obtained from Prof. Gardiner at Colorado University, Denver. It shows the normalized data from whole lysate hippocampus of Spanish Ts65Dn ten months mice treated with R04938581 or saline as control vehicle. Each sample is associated with three replicate spots of a five point dilution series. The dataset is divided into four classes of mice which are control group and Ts65Dn group mice treated with saline or R04938581. Information of failed and rescued learning classes are obtained from the protein expression response of 11 and 10 mice, respectively.

\subsection{Data Preprocessing}

Both datasets have one or more mice with missing values. The missing values are replaced with the average expression levels of the corresponding sample of that protein in the same class. For example, if one mouse is missing the first sample expression level information, it is replaced with the average value of first sample protein expression of other mice in the same class. In addition to replacing the missing parts, all measurements were normalized with Z-score normalization to prevent proteins with higher values influence on the classification result erroneously. With Z-score normalization as stated in Eq (1), mean of the scores is subtracted from each score and then divided into the standard deviation [21].

$$
Z=\frac{x-\mu}{\sigma}
$$

\subsection{Feature Selection}

Feature reduction is the process of reducing the number of features to identify the most relevant and important variables. It is very important as it helps us to understand information about the class of mice. It has the effect of decreasing the computational cost. Feature selection and feature integration methods are used for feature reduction. Feature selection selects a subset of features, while feature integration generates a new feature set of original features. Wrapper method is a type of the feature selection method and it uses a subset of features and train a model using them. One type of wrapper method, named as forward feature selection, is used in our work. It begins with an empty set and adds features based on classifier result. It does not examine all possible subsets and does not have a guarantee to find the optimal subset. However, it reduces the search time compared to exhaustive feature selection [22].

Using the Knostanz Information Miner (KNIME), forward feature selection is applied [23]. The idea is to program a search loop. Inside the loop, the dataset is partitioned into a learning set (70\%), used for the construction of the model in the current selection of the variables and a validation set (30\%), allowing for computing an unbiased estimation of the error rate. For learning process, Naive Bayes Learner is used and features are selected based on their effects of the improvement on our model. Scorer component computes 
confusion matrix and test error rate.

\section{Results}

Using the KNIME tool [23], forward feature selection is applied for obtaining the feature subsets of datasets which show us the critical proteins expressed in response to memantine or R04938581. Table 1 shows the expressed proteins when mice are injected with memantine and R04938581 drugs. Four gene products shown in bold are expressed in common with R04938581 and memantine treated Ts65Dn.

Table 1. Feature Subsets of Datasets

\begin{tabular}{ccc}
\hline \hline Feature No & Feature Subset & Feature Subset of Previous Work \\
\hline 1 & BRAF & BRAF \\
2 & S6 & S6 \\
3 & MEK & CDK5 \\
4 & ADARB1 & BDNF \\
5 & pBRAF & pCREB \\
6 & PKCA & PKCA \\
7 & NR1 & SOD1 \\
8 & SHH & PSD95 \\
9 & BDNF & pNR2A \\
\hline \hline
\end{tabular}

The selected proteins in these feature subsets have important roles in learning pathway and neural growth. BRAF, PKCA and MEK genes are associated with MAPK pathway and important in L/M process. Ribosomal Protein S6 and pGSK3B are components of mTOR pathway which takes action in learning. NR1 is the component of NMDAR receptor which plays an essential role in excitatory transmission and $\mathrm{L} / \mathrm{M}$ process. BDNF gene encodes a member of the nerve growth factor family of proteins The SHH gene provides instructions for making a protein called Sonic Hedgehog which is necessary for the development of forebrain. This signaling protein helps establish the line that separates the right and left sides of the forebrain.

\section{Conclusions}

In this paper, we have reported two different drug responses to protein expression in DS. The motivation of this research is to understand whether different drugs-treated Ts65Dn mice exhibit similar response or not. With the obtained result, the molecular pathway of rescued performance in DS can be understood and effective drug can be developed.

DS is the most prevalent genetic cause of ID in humans with an incidence of 1:700 in live births. Because of its high importance, understanding the cause of DS is required in order to develop drugs for rescuing learning. Thus, the critical roles of proteins which are expressed with different drugs and are potential targets of drugs have been analyzed by comparing protein expression levels of trisomic mice when learning is failed or rescued. The Ts65Dn group of mice fail to learn; this learning impairment can be corrected, however, if the Ts65Dn are injected with drug prior to training.

In previous works, using statistical analysis methods and machine learning techniques, drug responses of memantine and R04938581 are evaluated separately. In our work, protein subsets of two drug responses are evaluated together in order to understand general drug response and to find common pathway in which selected proteins in these two subsets are affected.

Before selecting features, preprocessing step consisted of filling missing values and normalization. These 
parts are carried out differently than previous works. Missing values are replaced with the average protein expression level of corresponding sample in the same class. For normalization, $\mathrm{Z}$ score normalization is applied to prevent influence of proteins with higher values on the classification outcome. In the next step, the forward feature selection method is applied to find most correlated and informative proteins. Forward feature selection is multivariate feature selection method in which features are handled together rather than one by one. For learning process, Naive Bayes classifier is used and features are selected based on their effects of the improvement on our model.

With forward feature selection technique, the critical factors in two datasets are determined. The results of selection method show that the selected proteins in two subsets have important roles in learning pathway and neural growth. In addition, there are common proteins which are expressed with both drugs and so it can be concluded that different drugs treated Ts65Dn mice exhibit similar responses. Abnormalities of these selected proteins may result in various symptoms of DS. Thus, these proteins and their interactions must be evaluated in pathway analysis to develop effective drugs for the treatment of DS. By giving efforts to selected subsets in our work, the rescued performance in DS can be understood and by analyzing the pathways in which selected proteins take action new drug design for the treatment of DS can be possible.

\section{Acknowledgments}

The authors would like to thank Katheleen J. Gardiner for providing us R04938581 drug response dataset.

\section{References}

[1] Sturgeon, X. J., \& Gardiner, K. (2011). Transcript catalogs of human chromosome 21 and orthologous chimpanzee and mouse regions. Mammalian Genome, 22, 261-71.

[2] Chapman, R. J., \& Hesketh, L. (2000). Behavioral phenotype of individuals with Down syndrome. Ment Retard Dev Disabil Res Rev, 6, 84-95.

[3] Silverman, W. (2007). Down syndrome: Cognitive phenotype. Mental Retardation and Developmental Disabilities Research Reviews, 13(3), 228-236.

[4] Nadel, L. (2003). Down's syndrome: A genetic disorder in biobehavioral perspective. Genes Brain Behav, 2(3), 156-66.

[5] Davisson, M. T. (1993) Segmental trisomy as a mouse model for Down syndrome. Prog. Clin. Biol. Res. 384, 117-133.

[6] Rueda, N., Florez, J., \& Martinez-Cue, C. (2012). Mouse models of Down syndrome as a tool to unravel the causes of mental disabilities. Neural Plast, 1-26.

[7] Gardiner, K. J. (2015). Pharmacological approaches to improving cognitive function in Down syndrome: current status and considerations. Drug Des. Devel. Ther., 9, 103-125.

[8] Braudeau, J. et al. (2011). Specific targeting of the GABA-A receptor $\alpha 5$ subtype by a selective inverse agonist restores cognitive deficits in Down syndrome mice. Journal of Psychopharmacology, 25(8), pp. 1030-1042.

[9] Martínez-Cué, C., Martínez, F. P., Rueda, N., Vidal, R., García-Cerro, S., \& Sánchez, et al. (2013). Reducing GABAA 5 receptor-mediated inhibition rescues functional and neuromorphological deficits in a Mouse model of down syndrome. The Journal of neuroscience, 33, 3953-3966.

[10] Costa, A. C., Scott-McKean, J. J., \& Stasko, M. R. (2008). Acute injections of the NMDA receptor Antagonist memantine rescue performance deficits of the Ts65Dn mouse model of down syndrome on a fear conditioning test. Neuropsychopharmacology: Official publication of the American College of 
Neuropsychopharmacology, 33, 1624-32.

[11] Chang, Q., \& Gold, P. E. (2007). Age-related changes in memory and in acetylcholine functions in the hippocampus in the Ts65Dn mouse, a model of Down syndrome. Neurobiology of Learning and Memory, 89(2), 167-77.

[12] Corrales, A., Martínez, P., García, S., Vidal, V., García, E., \& Flórez, J., et al. (2013). Long-term oral administration of melatonin improves spatial learning and memory and protects against cholinergic degeneration in middle-aged Ts65Dn mice, a model of Down syndrome. J. Pineal Res., 54, 346-358.

[13] Das I., Park J. M., \& Shin J. H., et al. (2013). Hedgehog agonist therapy corrects structural and cognitive deficits in a Down syndrome mouse model. Science Translational Medicine, 5(201), 201ra120.

[14] Busciglio, J., Capone, G., O’Bryan, J., \& Gardiner, K. J. (2013). Down syndrome: Genes, model systems, and progress towards pharmacotherapies and clinical trials for cognitive deficits. Cytogenet. Genome Res., 141, 260-271.

[15] Gardiner, K. L. (2009). Molecular basis of pharmacotherapies for cognition in down syndrome. Trends in Pharmacological Sciences, 31(2), 66-73.

[16] Chen, H. S., \& Lipton, S. A. (2005). Pharmacological implications of two distinct mechanisms of interaction of memantine with N-methyl-D-aspartate-gated channels. J Pharmacol Exp Ther, 314, 961-971.

[17] Lipton, S. (2007). Pathologically-activated therapeutics for neuroprotection: Mechanism of NMDA receptor block by memantine and S-nitrosylation. Current Drug Targets, 8, 621-32.

[18] Ahmed, M. M., Dhanasekaran, A. R., Block, A., Tong, S., Costa, A. C., Stasko, M., \& Gardiner, K. J. (2015). Protein dynamics associated with failed and rescued learning in the Ts65Dn mouse model of down syndrome. PloS One, 10(3), e0119491.

[19] Higuera, C., Gardiner, K. J., \& Cios, K. J. (2015). Self-organizing feature maps identify proteins critical to learning in a mouse model of down syndrome. PloS one, 10(6), e0129126.

[20] Dua, D., \& Karra, T. E. (2017). UCI machine learning repository. Irvine, CA: University of California, School of Information and Computer Science.

[21] Abdi, H., \& Williams, L .J. (2010) Normalizing data. Encyclopedia of Research Design, 935-938. Sage, Thousand Oaks.

[22] Jain, A. K., \& Douglas, Z. (1997). Feature selection: Evaluation, application, and small sample performance. IEEE Transactions on Pattern Analysis and Machine Intelligence, 19. 153-158.

[23] Meinl, T., Cebron, N., Gabriel, T., Dill, F., Kötter, T., \& Ohl, P., et al. (2007). The konstanz information miner 2.0. Open Source in Data Mining Workshop.

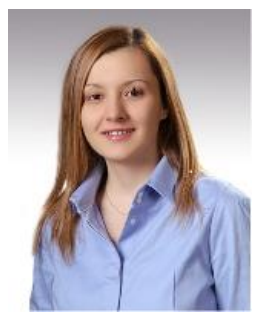

Handan Kulan was born in Turkey, in 1989. She received her B.S. degree from the Computer Engineering Department of Yeditepe University, Istanbul, Turkey in 2013. She received her M.S. degree from the Computer Science and Engineering Department of Sabancı University, Istanbul, Turkey in 2014. Currently, she is a PhD student at the Computer Engineering Department of Kadir Has University in Istanbul, Turkey. Her research interests include bioinformatics, big data and neuroscience.

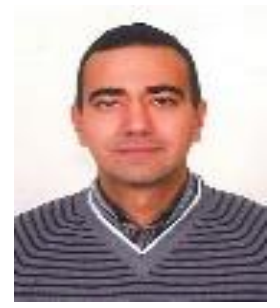

Tamer Dag was born in Turkey, in 1973. He received his B.S. degree from the Electrical and Electronics Engineering Department of Middle East Technical University, Ankara, Turkey in 1994. He received his M.S. and Ph.D. degrees from the Electrical and Computer Engineering Department of Northeastern University, Boston, MA, USA in 1997 and 2000 respectively. He worked as a research assistant at Northeastern University. Later he worked as a senior software engineer at Lucent Technologies in Westford, MA, USA. 
Currently, he is working at the Computer Engineering Department of Kadir Has University in Istanbul, Turkey. His research interests include computer networks, wireless sensor networks, network security, indoor positioning systems and bioinformatics.

Prof. Tamer Dag is a member of IEEE and has played an active role for the organization of some IEEE conferences. He has reviewed many conference and journal papers in his fields of expertise. 